\title{
Self-oscillations of non-neutral plasma diode
}

\section{Levko}

${ }^{1}$ CFD Research Corporation, Huntsville, AL 35806, USA

(Received: 23. May 2020, Accepted: 16. Dec. 2020, Published online: 17. Dec. 2020)

Self-oscillations of non-neutral plasma diode operating in the anode-glow mode are analysed using the self-consistent one-dimensional Particle-in-Cell Monte Carlo collisions model. In order to obtain these states, the current exceeding the space-charge limited current has to be emitted from the cathode, the electron mean free path must be much longer than the cathode-anode gap, and the cathode voltage must be slightly larger than the ionization potential of the background gas. It is obtained that in such a case, immobile ions form the electrostatic trap for the electrons generated in the cathode-anode gap. These electrons oscillate between the cathode and the anode causing the self-oscillations of the plasma potential. It is shown that the increase of the emission current leads to the increase of the frequency of the obtained self-oscillations. Starting at some value of the emission current, a lot of the emitted electrons are got trapped in the electrostatic well, which leads to the transition to chaos.

(C) G-Labs 2020

(DOI: 10.31281/jtsp.v1i1.12)

dima.levko@gmail.com

\section{Introduction}

Discharges driven by electron emission from the walls have long history [1] and are of interest for many applications such as electric propulsion, plasma etching, high-power microwaves generation etc. [2]. Depending on the parameters such as emission current, background gas pressure and gas component content, and electrodes configuration, different modes of discharge are obtained. The type of discharge also depends on the electron emission mechanism. Thermionic and field electron emitters are two most common types of cathodes used today.

Initially, the interest to discharges ignited by thermionic electron emission was driven by arc discharges [3]. Later, these discharges have found their application for thermionic energy conversion to electricity. [4] During the last three decades, a lot of research is devoted to the studies of microwaves generation from diodes with the electron emission from the walls $[5,6,7]$. Also, thermionic emission drives more complex setups for diamond film deposition, [8] or hollow cathodes for electric propulsion. [9]

Both experimental and theoretical research studies have shown that these discharges exhibit interesting properties especially at low gas pressures $[10,11,12]$. These are the virtual cathode formation, double layers, various types of instabilities, transition to chaos etc. Non-linear oscillations obtained in Q-machines were investigated in numerous papers (see Refs. $[13,14]$ and references therein). In these papers, both Particle-in-Cell (PIC) and fluid codes were used. Both electrons and ions were injected from the diode walls and were considered as collisionless fluids. This is so-called Knudsen regime in which ions are generated only at the anode surface. It was obtained that the diode states depend on two parameters: 1) the ratio between the cathode-anode gap voltage and the electron temperature, and 2) the ion-to-electron density ratio.

A series of papers $[10,11,15]$ was devoted to the analysis of chaotic states of volume discharges driven by thermionic emission. In these papers, the one-dimensional PIC Monte Carlo collisions 
(1D PIC/MCC) models were used. The plasma was generated self-consistently by the background gas ionization. Three states of the diode were obtained. These are the anode glow mode, the temperature limited mode and the double layer mode. More recently, these states were examined by the Vlasov-Poisson equations model [16].

In the anode glow mode, the gas ionization occurs only in the vicinity of the anode [12]. The emitted current is such that it is ruled by the space charge limit, i.e. the virtual cathode is formed in the vicinity of the cathode and reflects most of the emitted electrons. In the temperature-limited mode, the gas is ionized in the entire domain except the cathode and the anode sheaths which are usually collisionless at the operating conditions [12]. The authors of Ref. [12] showed that the increase of the cathodeanode (CA) gap voltage results in the increase of the density of plasma generated. This leads to the expansion of the anode glow toward the cathode and the anode glow mode finally transits into the temperature-limited mode. They also obtained useful expression which allows one to calculate the length of the anode glow as the function of the CA gap, type of gas and applied voltage (see discussion in Section II below).

As it follows from the results presented, for instance, in Refs. $[10,11,12]$, the plasma is nonneutral in the anode glow mode. This plasma consists of the regions with excess of either negative or positive space charge. For long gaps $(10-20 \mathrm{~cm})$, analysed in these references, the moving virtual cathode is obtained. As it follows from the analysis presented in Ref. [16], this happens because the plasma ions experiencing charge exchange collisions with neutrals are got trapped in the virtual cathode. This compensates the negative space charge of the virtual cathode and leads to its shift toward the anode. However, one might ask: what will happen with the anode glow mode for gaps much shorter than the ion mean free? The answer on this question is not so trivial. For instance, recently, Pramanik et al. [17] have shown using one-dimensional fluid model that the accounting for weak electron-neutral collisions generates new family of solutions for non-neutral plasma diodes.

The main goal of the present paper is to answer the question asked above. Here, the selfconsistent one-dimensional Particle-in-Cell Monte Carlo collisions (1D PIC/MCC) model is used for analysis. The electrons are injected from the cathode having half-Maxwellian velocity distribution function. The plasma is generated by these electrons as well as by the electrons generated in the CA gap due to the background gas ionization.

\section{Numerical model parameters and problem formulation}

The 1D PIC/MCC model [18] used in the present paper resolves three velocity components of both electrons and ions and one spatial component. This code is similar with the one used in Refs. $[10,12]$ for the modelling of discharge driven by thermionic emission for higher gas pressure. The model considers only two plasma species, electrons and singly charged ions. The space step was chosen equal to $10^{-5} \mathrm{~m}$, while the time step was $10^{-12} \mathrm{~s}$ in order to satisfy the Courant condition.

The working gas is helium at the pressure of 0.3 $\mathrm{Pa}$ and the temperature of $300 \mathrm{~K}$ (the gas density is $n_{g}=7.2 \times 10^{19} \mathrm{~m}^{-3}$ ). The choice of pressure is discussed below. The CA gap is $d_{\text {gap }}=1 \mathrm{~cm}$. The left electrode, cathode, is at the constant potential of $\varphi_{C}=-40 \mathrm{~V}$, while the right electrode, anode, is grounded. The model takes into account the ion-neutral collisions (both charge exchange and elastic scattering) using the cross sections from Phelps database [19]. Note that these collisions can be neglected in the present studies because the ion-neutral collision mean free path is much longer than the CA gap. Indeed, the typical ion energy in the vicinity of the cathode is $\sim 1-10 \mathrm{eV}$. The charge-exchange cross section for such ions is $\sigma_{e x} \sim 2 \times 10^{-19} \mathrm{~m}^{2}$. Then, the ion mean free path at the gas pressure of $0.3 \mathrm{~Pa}$ is estimated as $\lambda_{i o n}=\frac{1}{n_{g} \sigma_{e x}} \sim 7 \mathrm{~cm}$.

The cathode temperature is an external parameter which is varied in the range 1800-2050 $\mathrm{K}$. The electron emission from the wall is described by the Richardson equation:

$$
j_{e m}=D T_{C}^{2} \exp \left(\frac{-q_{e} \varphi_{0}}{k_{B} T_{C}}\right) .
$$

Here $D=1.2 \times 10^{6} \mathrm{~A} / \mathrm{m}^{-2} \mathrm{~K}^{-2}$ is the constant whose value, in general, is defined by the emitter material, $q_{e}$ is the elementary charge, $\varphi_{0}=4.5 \mathrm{eV}$ is the emitter work function, $k_{B}$ is the Boltzmann constant, and $T_{C}$ is the cathode temperature. For simplicity of analysis, the Schottky effect, i.e. the influence of electric field on the emission current, was not considered here.

For the simplicity of analysis, the electrons emitted from the cathode and electrons generated in the CA gap are considered as two separate species, namely, emitted $\left(n_{\text {emm }}\right)$ and 
plasma $\left(n_{e}\right)$ electrons. They have the same properties and experience the same collisions but are treated as two different kinds of computational particles. Below, the plasma electrons are also called as generated electrons.

Three electron-neutral collisions are considered in the model. These are the excitation of the first electronic level of $\mathrm{He}$, momentum transfer collision and ionization. The latter process leads to the generation of electron/ion pairs in the gap. The cross sections are taken from the Biagi's database [20]. The electron-neutral collisions were modeled using the methods described, for instance, in Ref. [21].

Now, let us discuss the choice of the initial conditions. As was obtained in Ref. [12], the length of the anode glow in the anode-glow regime of the discharge driven by thermionic emission is

$$
l \approx d_{\text {gap }} \cdot\left(\frac{\left|\varphi_{C}\right|-\varepsilon_{\text {ion }}}{\left|\varphi_{C}\right|}\right) .
$$

Here, $\varepsilon_{\text {ion }}$ is the ionization threshold of $\mathrm{He}\left(\varepsilon_{\text {ion }} \approx\right.$ $24.6 \mathrm{eV})$. Then, for $\varphi_{C}=-40 \mathrm{~V}$ one has $l \approx$ $0.44 \cdot d_{\text {gap }}$. The mean free path of electrons in $\mathrm{He}$ gas at the pressure of $0.3 \mathrm{~Pa}$ can reach $100 \mathrm{~cm}$, which is much longer than the CA gap. This means that only a few electrons experience the collisions with neutrals. However, as will be seen below, this is enough for the generation of rather dense plasma. Also, the electrons being generated in the gap due to the gas ionization will escape to the anode without any collisions. The escape of electrons from the gap leaves behind uncompensated positive space charge which creates the potential well for electrons.

As it follows, for instance, from Ref. [12], the discharge driven by thermionic emission in $\mathrm{He}$ can be supported by the voltage as low as $U_{d} \sim 25$ $\mathrm{V}$, which is comparable with the He ionization threshold. This means, if we choose the value of the discharge voltage slightly larger than $U_{d}$, the electrons being generated in the gap will be captured by the ion potential well. Since the mean free path of generated electrons is much longer than $d_{\text {gap }}$, they will oscillate in the well until some stabilization mechanism is established. Below, such mechanisms are reported.

\section{Results and discussion}

Below, the results of simulations obtained for the cathode temperature in the range 2000-2125 K are presented (see Figs. 1-9). The emission current was varied in the range 21-110 A/m , which, as it follows from the Child-Langmuir law, exceeds the vacuum space-charge limiting current $\left(j_{C L}\right)$ of the considered diode. For the conditions of the present studies, this current is $\sim 5.9 \mathrm{~A} / \mathrm{m}^{2}$. The simulation results have shown that for $T_{C}<2000 \mathrm{~K}$, the stable discharge is obtained. In this regime, the cathode sheath occupied almost entire domain, while the quasineutral plasma was obtained only in the vicinity of the anode. The formation of this plasma suppressed the virtual cathode formation, which is obtained for the plasma-free gap. This occurred because the quasi-neutral plasma acts as the virtual anode. This reduced the gap in which the electrons are emitted, which leads to the increase of the space-charge limiting current. The stable plasma obtained for $T_{C}<2000 \mathrm{~K}$ is not of interest here.

For $T_{C}>2150 \mathrm{~K}$, the column of quasi-neutral plasma also establishes between the electrodes. The plasma density in this column reaches the value $\sim 10^{10} \mathrm{~cm}^{-3}$, and this plasma occupies almost entire domain except narrow region near the cathode (cathode sheath). Its thickness is $\sim 0.01$ $\mathrm{cm}$, which is much shorter than the electron mean free path. That is, the cathode sheath is collisionless. The emitted electrons form almost monoenergetic beam while propagating through this sheath. Depending on the emission current and the density of plasma generated between the cathode sheath and the anode, the plasma is either stable or unstable with respect to the excitation of two-stream instability. This regime was analysed in previous publication [22] and is not of interest here.

For the intermediate emitter temperatures ( $\left.T_{C}=2000-2125 \mathrm{~K}\right)$, the generation of non-neutral plasma in the CA gap was obtained. Figs. 1-3 show the results of simulations obtained for the emitter temperature of $2000 \mathrm{~K}$. One can conclude that near the anode, the ion density exceeds the electron density (i.e. $n_{e m m}+n_{e}$ ), while near the cathode, the dominant species are the electrons, and $n_{e m m}>n_{e}$. Fig. 1(c) shows that there is no virtual cathode formation. This means that all of the emitted electrons enter the interelectrode space. One can also see that the plasma potential with respect to the anode is $\sim 7 \mathrm{~V}$. 

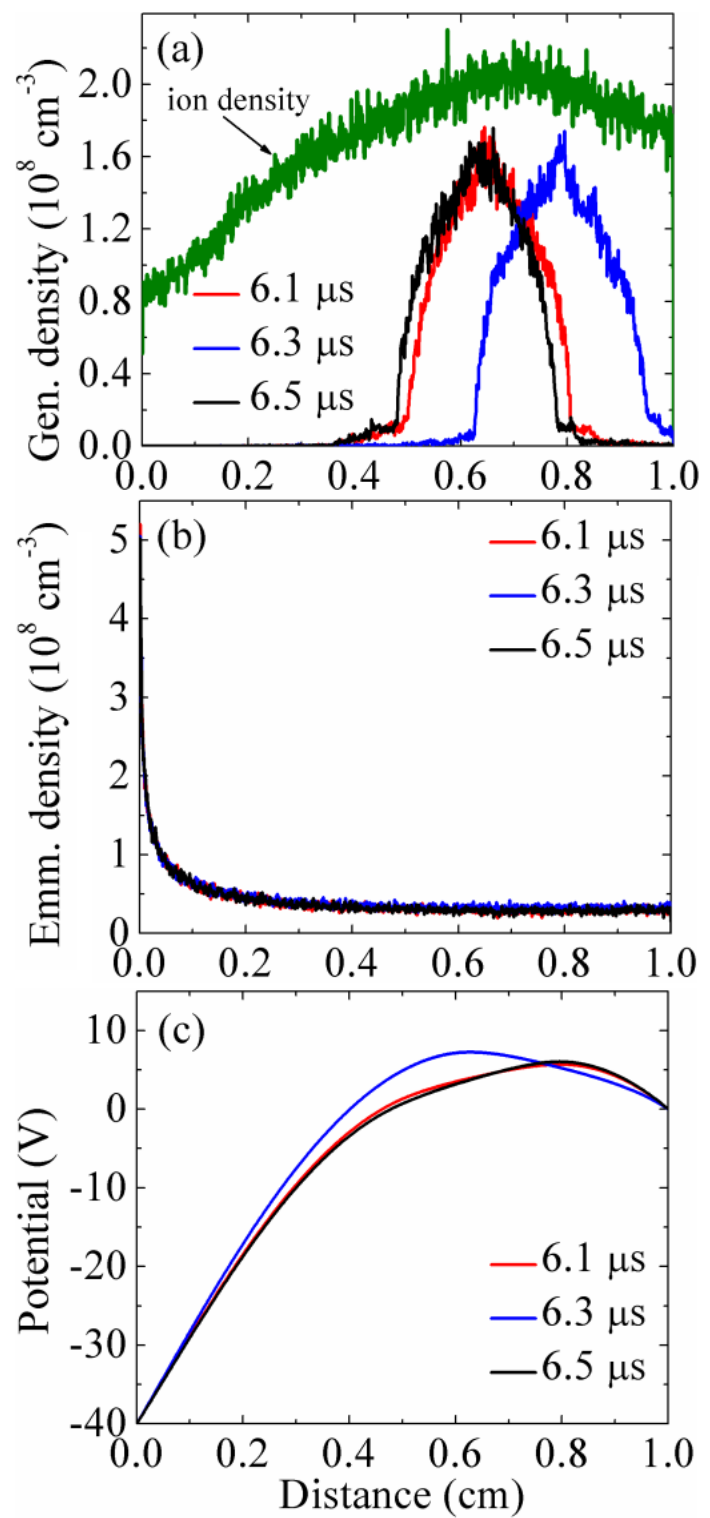

Figure 1. One-dimensional profiles of (a) the density of electrons generated in the cathodeanode gap, (b) the density of electrons emitted from the cathode, and (c) the potential. The cathode temperature is $2000 \mathrm{~K}$.

The 1D profiles of the densities of generated and emitted electrons are shown in Fig. 1(a) and Fig. 1 (b), respectively. Fig. 1(a) also shows the ion density. It is seen that in the region $0.4 \mathrm{~cm}<x<1$ $\mathrm{cm}$, the density of generated electrons is $\sim 3$ times larger than the density of emitted electrons. Thus, the plasma potential seen in Fig. 1(c) is defined by the ions and the generated electrons. Namely, the potential trough is formed in such a way in order to capture the generated electrons and prevent their escape to the anode. Fig. 1(a) shows that the plasma electrons oscillate between the anode and the centre of the simulation domain, while the density of emitted electrons remains almost constant.
These oscillations are better seen in Fig. 2, which shows the space-time diagrams of the density of generated electrons and potential. One can see that both oscillate with the frequency $v_{0} \sim 0.1 \mathrm{GHz}$ in spite of the constant applied voltage. The same oscillations were obtained for the electric field (not shown here). At the same time, the densities of emitted electrons and ions do not vary in time. For ions, this is obtained because they are massive and do not respond the electric field oscillating with the $\mathrm{GHz}$ frequency. The emitted electrons do not respond the oscillating electric field because their time-of-flight through the gap, $\tau_{\text {emm }}$ is much shorter than the period of oscillations. Indeed, one can estimate this time as $\tau_{\text {emm }} \sim d_{\text {gap }} / v_{\text {emm }}$, where $v_{\text {emm }}$ is the average velocity of emitted electrons. Using the values $d_{\text {gap }}=1 \mathrm{~cm}$ and $v_{\text {emm }} \sim 4 \times 10^{6} \mathrm{~m} / \mathrm{s}$ (see phase space in Fig. 3(a)), one estimates $\tau_{\text {emm }} \sim 2.5$ ns, i.e. $1 / \tau_{\text {emm }} \sim 0.4 \mathrm{GHz}$, which is $\sim 4$ times larger than $v_{0}$. Thus, the emitted electrons cross the CA gap much faster than the period of self-oscillations.

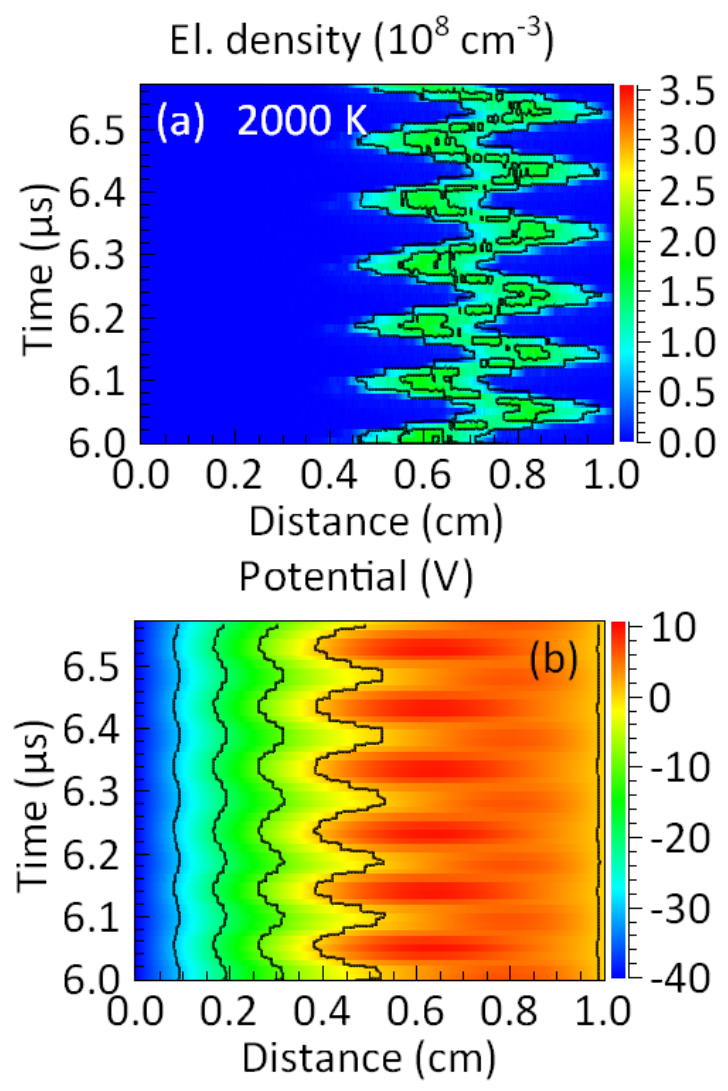

Figure 2. Space-time diagram of (a) electron density and (b) potential obtained for the cathode temperature of $2000 \mathrm{~K}$.

The time dependence of the potential obtained at three different locations of the CA gap is shown in Fig. 8(a). The Fast Fourier transform (FFT) of the potential obtained in the centre of the domain is shown in Fig. 9. Fig. 8(a) shows that the frequencies of potential oscillations at $0.25 \times d_{\text {gap }}$ 
and $0.5 \times d_{\text {gap }}$ are the same $(\sim 0.1 \mathrm{GHz}$, see Fig. 9). However, the signal changes at the distance of $0.75 \times d_{\text {gap }}$ from the cathode. One can see the generation of the second harmonic at the frequency of $\sim 0.2 \mathrm{GHz}$.
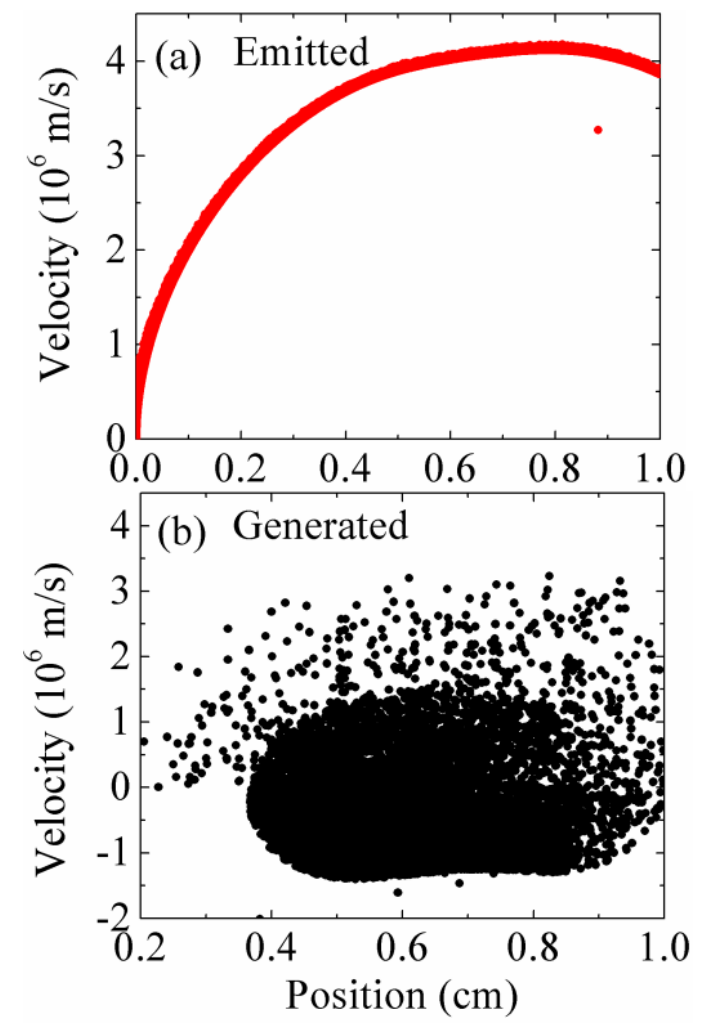

Figure 3. Phase space of (a) emitted electrons, (b) electrons generated in the cathode-anode gap. The cathode temperature is $2000 \mathrm{~K}$.

The nature of the obtained self-oscillations can be understood, using the information about the electron motion at the given conditions. The phase spaces of emitted and plasma electrons (the velocity component only in the simulated direction) are shown in Fig. 3. One can conclude from Fig. 3(a) that the emitted electrons are not trapped in the CA gap. The main part of the emitted electrons does not experience any inelastic collisions with neutrals. They leave the CA gap in the form of beam. The velocity spread of this beam is caused by the velocity modulation by the oscillating potential in the vicinity of the cathode.

As it follows from Fig. 3(b), the main part of the plasma electrons is trapped in the CA gap. They form the core oscillating between the cathode and the anode sheaths. The energy of only a few of these electrons exceeds the ionization threshold of $\mathrm{He}$ atom. Therefore, the plasma electrons contribution to the plasma generation is negligible. The simulation results show that the average velocity of plasma electrons is $v_{p l} \sim 3 \times 10^{5}$ $\mathrm{m} / \mathrm{s}$.
The dominant electron-neutral collision for the trapped electrons is the momentum transfer. In $\mathrm{He}$, the cross section of this collision is almost constant for $\varepsilon_{e}<10 \mathrm{eV}$ and is equal to $\sigma_{m} \sim 5 \times 10^{-}$ ${ }^{20} \mathrm{~m}^{2}$. [20 Then, the mean free path of trapped electrons is estimated as $\lambda=\frac{1}{n_{g} \sigma_{m}}$. At the pressure of $0.3 \mathrm{~Pa}$, it is $\sim 28 \mathrm{~cm}$, i.e. $\lambda \gg d_{\text {gap }}$. Since in each momentum transfer collision electron loses only a small fraction of its energy, electron being generated in the gap oscillates between the anode and the cathode sheaths with negligibly small energy losses. When the core of generated electrons moves toward the anode, it modifies the plasma potential (see Fig. 1(c)). Then, when the electrons approach the anode, the potential near the anode slightly decreases and some fraction of plasma electrons leaves the potential well. The electron escape leads to the recovery of the anode sheath voltage which stops the electron escape to the anode. Then, the electrons which remain in the CA gap reflect from the anode sheath and move toward the cathode. The cathode sheath reflects these electrons because the cathode sheath voltage is much larger than the trapped electrons energy. While the reflected electrons move back to the anode, a few electrons are generated by the emitted electrons. When these electrons reach the anode, they modify the anode sheath potential distribution. Then, most energetic of the trapped electrons escape to the anode, etc. This process, as it follows from Fig. 2, is periodic. Self-oscillations in the Knudsen-type diode having the same nature were analysed in Ref. [23].

Keeping this explanation in mind, it is possible to estimate the period of self-oscillations as the time-of-flight of the plasma electrons from the cathode to the anode. This time is estimated as $\tau_{p l} \sim d_{g a p} / 2 v_{p l}$. Here, $v_{p l} \sim 3 \times 10^{5} \mathrm{~m} / \mathrm{s}$ is the average velocity of plasma electrons, and $d_{\text {gap }} / 2$ takes into account that the electron core travels only a fraction of the CA gap. Then, one finds $1 / \tau_{p l} \sim 0.06 \mathrm{GHz}$, which is in rather good agreement with the results of present simulations (see, for instance, Fig. 9).

It was obtained that the self-oscillations disappear, if one increases the cathode potential. This result was also obtained in Ref. [12] and was identified as the transition from the anode-glow mode to the temperature-limited mode. This is also demonstrated in Fig. 4 which shows the results obtained for $\varphi_{C}=-80 \mathrm{~V}$. Other parameters were the same as for $\varphi_{C}=-40 \mathrm{~V}$. One can see the generation of the column of stable quasi-neutral plasma. 
The simulation results have shown that the increase of the emitter temperature from $2000 \mathrm{~K}$ to $2125 \mathrm{~K}$ results in the increase of the oscillation frequency (see Fig. 9). Fig. 9 shows narrow peaks on the FFT spectra in this range. At $T_{C}=2125 \mathrm{~K}$, the FFT spectrum broadens and establishes the properties of the chaotic behaviour [24]. One can conclude from Fig. 9 that the mechanism to chaos transition obtained in the present studies for the anode-glow mode differs from the one obtained in Refs. $[10,11,12]$. In those studies, the transition to chaos was obtained through the period doubling. In order to understand how the transition to chaos happens at the conditions of present studies, let us analyse the plasma dynamics at $T_{C}=2125 \mathrm{~K}$.
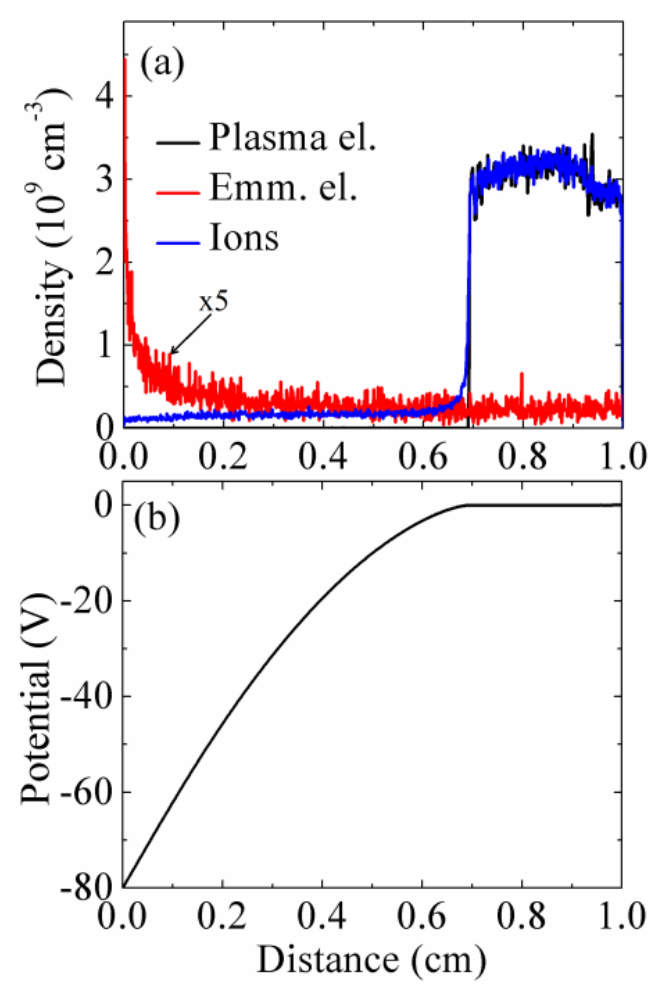

Figure 4 . The results of simulations obtained for the cathode potential of $-80 \mathrm{~V}$ : (a) densities, and (b) potential.

Fig. 5 shows the space-time diagram of the density of generated electrons and the potential for $T_{C}=2125 \mathrm{~K}$. The 1D profiles of the electron densities and potential at three different times are shown in Fig. 6. One can distinguish several features which are not obtained for $T_{C}=2000 \mathrm{~K}$. For instance, several spikes are seen on the spatial profile of the density of the emitted electrons (see Fig. 6(b)). This means the emitted electrons form the bunches. Note that the density in these bunches is comparable with the density of generated electrons, i.e. the influence of emitted electrons on the plasma potential increases for increasing emission current. The peak of plasma potential is $\sim 100 \mathrm{~V}$, which is almost 1 order of magnitude larger than that obtained for $T_{C}=2000 \mathrm{~K}$ (compare Fig. 6(c) and Fig. 1(c)).

The increase of the plasma potential for increasing emission current is obtained because the latter results in the increase of the plasma density generated in the gap. Since the plasma is still non-neutral for $T_{C}=2125 \mathrm{~K}$, the increase of the ion density results in the increase of the plasma potential.

The typical phase spaces of the emitted and plasma electrons are shown in Fig. 7. One can see that there are a lot of generated electrons having the energies exceeding the ionization threshold of He. The simulation results have shown that for $T_{C}=2125 \mathrm{~K}, \sim 57 \%$ of plasma is generated by the emitted electrons and $\sim 43 \%$ is generated by the plasma electrons, i.e. the contribution of both groups becomes comparable. Such energetic plasma electrons are explained by the fact that at $T_{C}=2125 \mathrm{~K}$ the depth of the potential well is $\sim 100$ V. Therefore, the plasma electrons get enough energy for the gas ionization during their propagation through the cathode sheath.

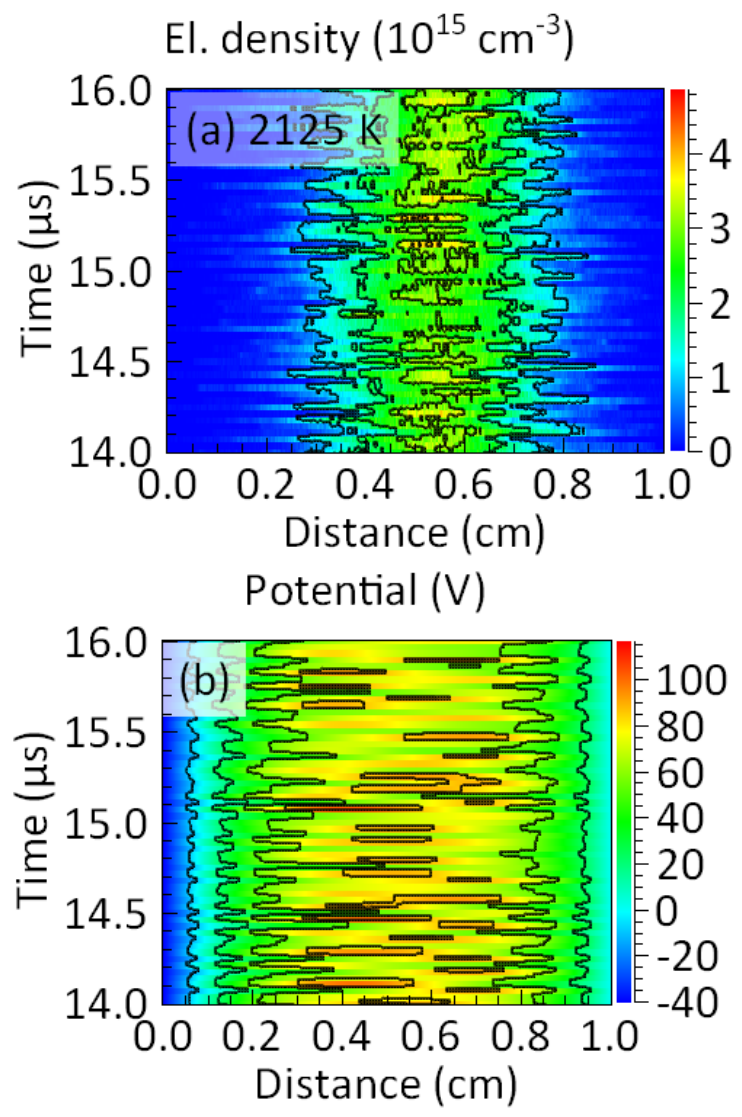

Figure 5. Space-time diagram of (a) electron density and (b) potential obtained for the cathode temperature of $2125 \mathrm{~K}$. 

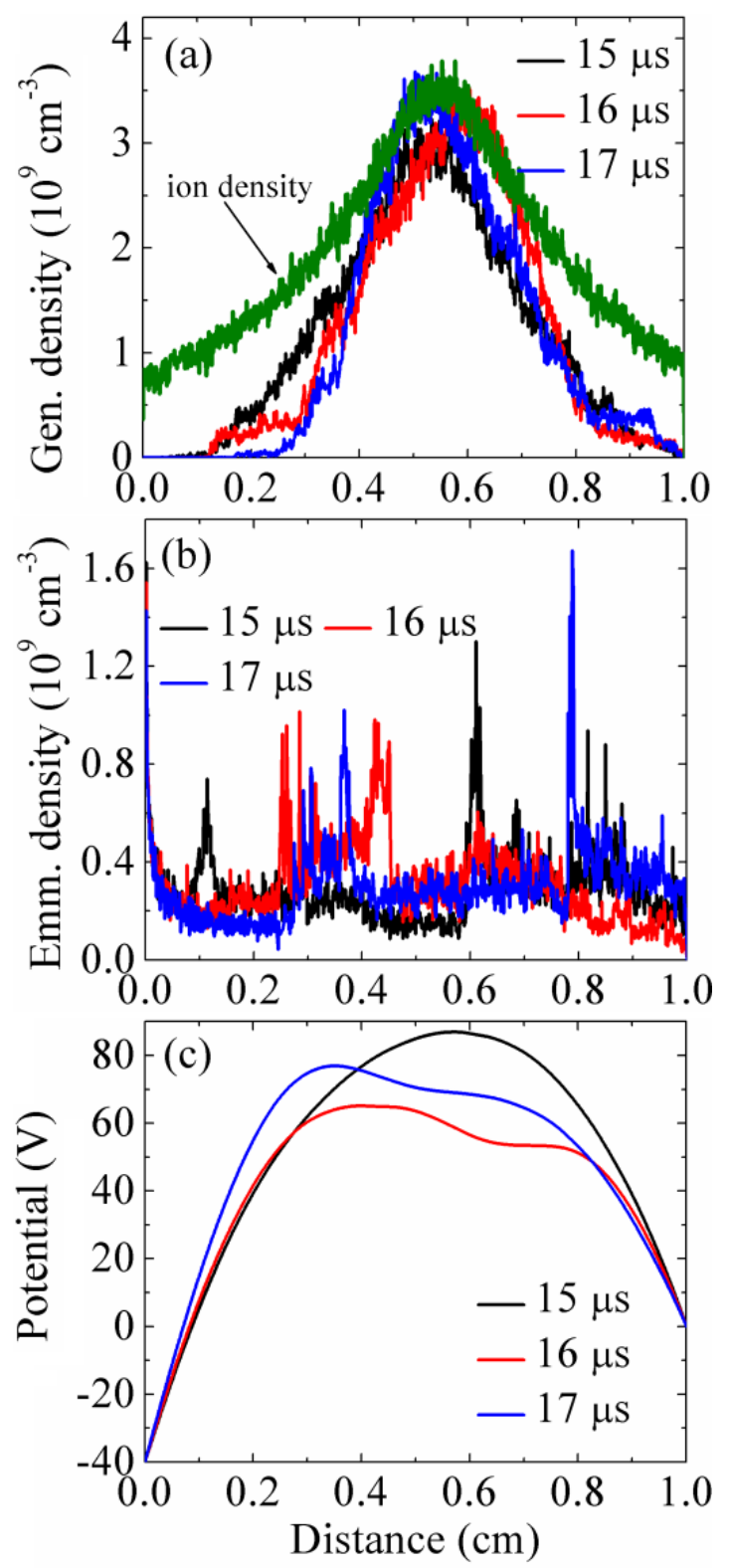

Figure 6. One-dimensional profiles of (a) emitted electrons density, (b) density of electrons generated in the cathode-anode gap, and (b) potential. The cathode temperature is $2125 \mathrm{~K}$.

The detailed analysis of the electron motion in the electric field in the vicinity of the cathode can be found, for instance, in Ref. [25]. It is shown that if the amplitude of the oscillating component of the electric field becomes comparable with its constant component, emitted electrons start reacting to the field oscillations. For some values of initial phase, the emitted electrons get decelerating and form bunches. This is obtained for $\mathrm{T}_{\mathrm{C}}=2125 \mathrm{~K}$.
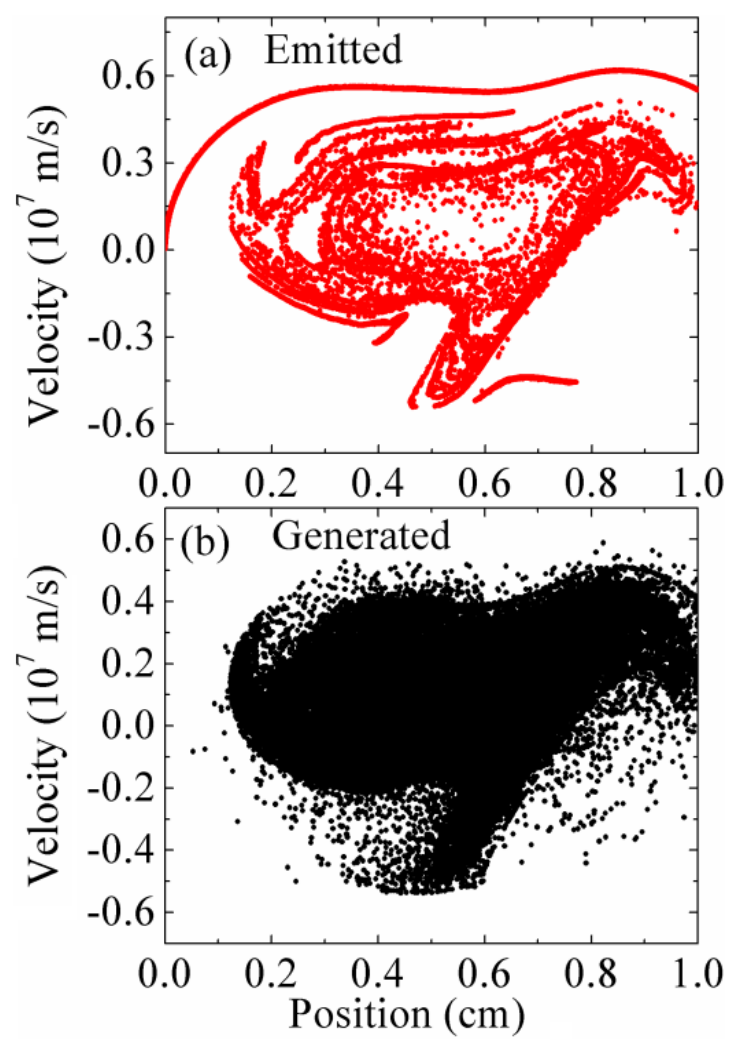

Figure 7. Phase space of (a) emitted electrons, (b) electrons generated in the cathode-anode gap. The cathode temperature is $2125 \mathrm{~K}$.

This conclusion is also supported by the results of present studies. Fig. 9 shows strong harmonic of the electric field having the frequency $\sim 0.45 \mathrm{GHz}$. The period of oscillations at this frequency is comparable with the time-of-flight of emitted electrons through the gap. This means the emitted electrons respond the oscillating field. If these electrons are emitted in phase with the oscillating field, they are kept accelerating while they propagate to the anode. However, if the electrons are emitted out of phase, they gain smaller velocity during the half period of oscillations. Some of these electrons have the energy smaller than the depth of the potential well and are got trapped in the potential well of ions. The bunching of these electrons is seen in Fig. 6(b).

Thus, one can conclude the following. The increase of $j_{e m}$ results in the increase of the ion density, which leads to the increase of both the plasma potential and the time-of-flight of plasma electrons. This leads to the increase of the oscillation frequency of the plasma potential and increase of the oscillating component of the electric field. At it follows from the electron trajectory analysis, at high oscillation frequencies of the electric field, emitted electrons start responding the oscillating field. The electrons, emitted out of phase of the electric field, form 
bunches which influence the distribution of electrostatic potential. The slowing down of the emitted electrons generates additional harmonics seen in Fig. 9(b). The contribution of the emitted electrons into the plasma potential also influences the trajectories of plasma electrons, which also leads to the generation of additional harmonics.
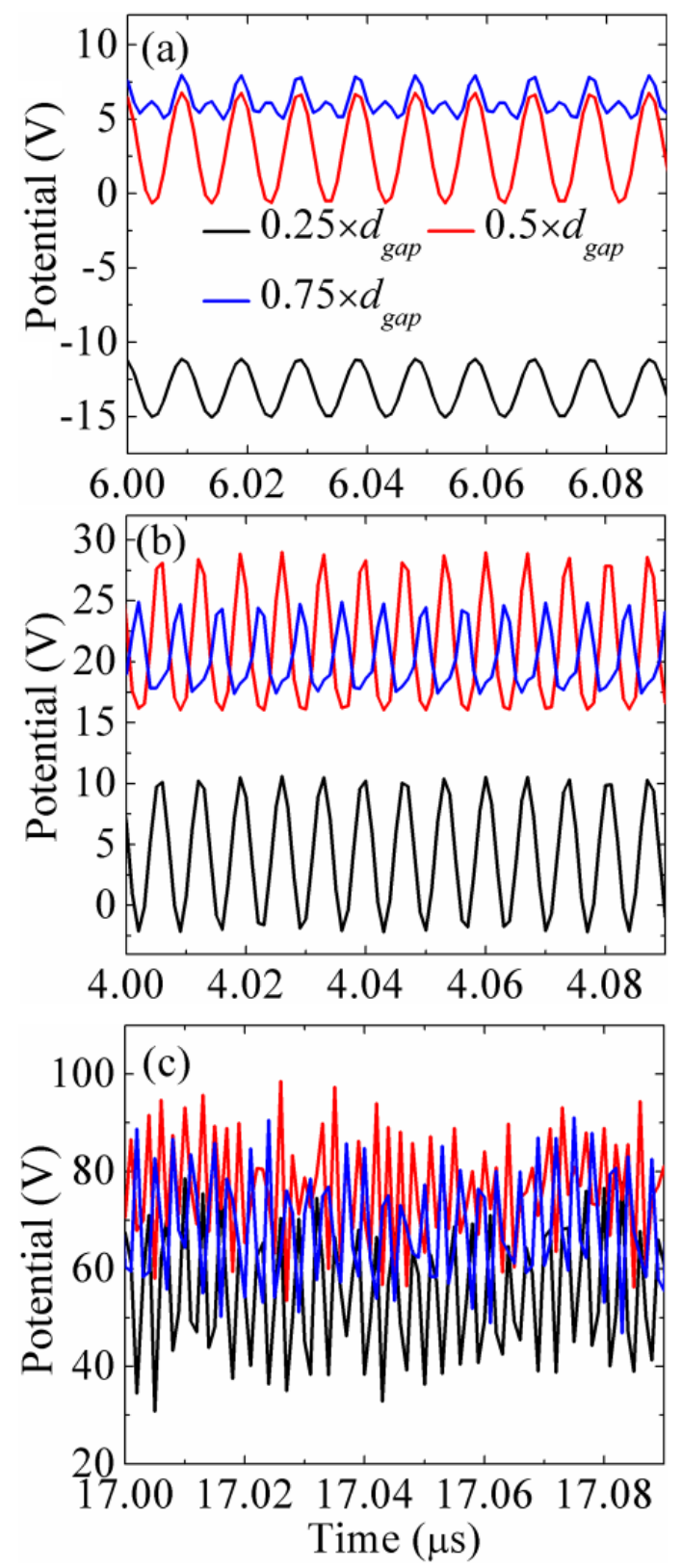

Figure 8. Time dependence of the potential obtained at three different locations of the cathode-anode gap for the emitter temperature of (a) 2000 K, (b) $2075 \mathrm{~K}$, and (c) $2125 \mathrm{~K}$.

The growing coupling between the plasma and emitted electrons through the plasma potential allows one to make the analogy between the diode considered in the present paper and the controlled nonlinear oscillator [24]. It is know that if the external force is controlled by motions of the oscillator itself, this system allows the transition to chaos through intermittency [24].

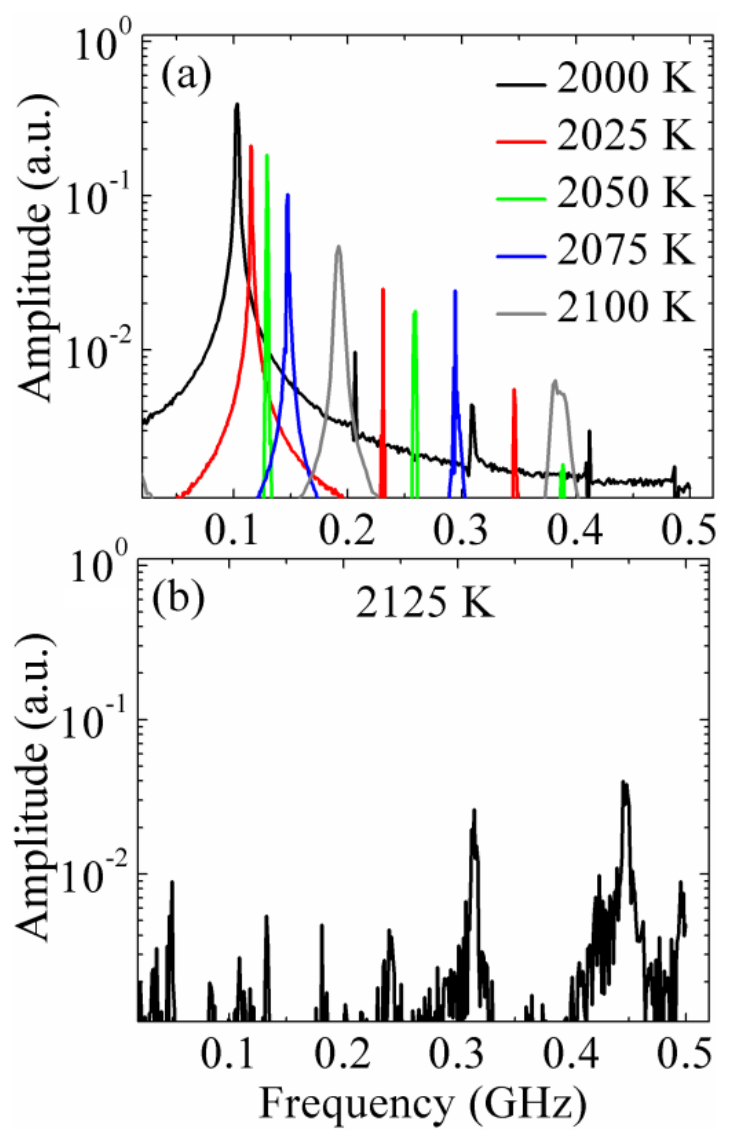

Figure 9. Fast Fourier transform of the potential obtained in the center of the cathode-anode gap (a) $T_{C}=2000-2100 \mathrm{~K}$, and (b) $2125 \mathrm{~K}$.

It is important to note that while in the vacuum gaps with intense electron beams chaotic and periodic regimes change each other, 6 this is not obtained for the conditions of the present studies. The simulation results have shown that further increase of the emission current results in the increase of the density of generated plasma. At some temperature, this plasma becomes quasi-neutral. Then, the plasma diode states reported in this paper disappear and other regimes of discharge operation are established [22].

\section{Conclusions}

Self-oscillations of the non-neutral plasma diode were analysed using the self-consistent onedimensional Particle-in-Cell Monte Carlo collisions model. These states are obtained for the emitter current in the range 21-110 $\mathrm{A} / \mathrm{m}^{2}$. Below this range, the diode is stable, while for higher currents the quasi-neutral plasma is generated.

The analysis has shown that the diode self- 
oscillations are obtained if the following three conditions are satisfied: 1) the space-charge limiting current is emitted from the cathode; 2) the electron mean free path is much longer than the cathode-anode gap; and 3) the cathode potential only slightly exceeds the ionization potential of the background gas. The selfoscillations were induced by the electron oscillations between the cathode and the anode sheaths in the potential well created by the ions.

It was obtained that the increase of the emission current leads to the increase of the frequency of self-oscillations. This led to the bunching of the emitted electrons, which finally led to the transition to chaos.

\section{References}

[1] I. Langmuir, Phys. Rev. 33, 954 (1929).

[2] E. Oks, Plasma Cathode Electron Sources (Wiley-VCH, 2006).

[3] R. Tanberg, Phys. Rev. 35, 1080 (1930).

[4] G. N. Hatsopoulos and E. P. Gyfopoulos, Thermionic Energy Conversion (MIT Press, Boston, MA, USA, 1973).

[5] Modern Microwave and Millimeter-Wave Power Electronics, edited by R. J. Barker, J. H. Booske, N. C. Luhmann Jr., G. S. Nusinovich (Wiley-IEEE Press, 2005).

[6] D. I. Trubetskov, A. E. Hramov, Lectures on High Frequency Electronics, Vol. 1 (Moscow, Fizmatlit, 2003) (in Russian).

[7] Y. Bliokh, J. Felsteiner, and Ya. Z. Slutsker, J. Appl. Phys. 111, 013302 (2012).

[8] J. Gruenwald, J. Reynvaan and P. Knoll. Phys. Scr. T161, 014006 (2014).

[9] D. Goebel, I. Katz, Fundamentals of Electric Propulsion (John Wiley \& Sons, 2008).

[10] F. Greiner, T. Klinger, and A. Piel, Phys. Plasmas 2, 1810 (1995).

[11] F. Greiner, T. Klinger, and A. Piel, Phys. Plasmas 2, 1822 (1995).

[12] H. J. Lee and J. K. Lee, Phys. Plasmas 5, 2878 (1998).

[13] F. Bauer and H. Schamel, Physica D 54, 235 (1992).
[14] K. H. Pae and S. J. Hahn, J. Phys. Soc. Jpn. 71, 2169 (2002).

[15] H. Kolinsky, F. Greiner and T. Klinger, J. Phys. D: Appl. Phys. 30, 2979 (1997).

[16] M. D. Campanell and M. V. Umansky, Plasma Source Sci. Technol. 26, 124002 (2017).

[17] S. Pramanik, V. I. Kuznetsov, and N. Chakrabarti, Phys. Plasmas 25, 083512 (2018).

[18] D. Levko, J. Appl. Phys. 114, 223302 (2013).

[19] Phelps database for $\mathrm{He}+$ - He collisions: www.Ixcat.net/Phelps

[20] Biagi database for electron - He collisions: www.Ixcat.net/Biagi-v7.1

[21] Z. Donko, Plasma Sources Sci. Technol. 20, 024001 (2011).

[22] D. Levko and L. L. Raja, Phys. Plasmas 23, 032107 (2016).

[23] V. I. Kuznetsov, A. Ya. Ender, S. Kuhn, Tech. Phys. 51, 1257 (2006).

[24] H. D. Abarbanel, M. I. Rabinovich, M. M. Sushchik, Introduction to Nonlinear Dynamics for Physicists (World Scientific Publishing, 1993).

[25] G. Nusinovich, M. Read, and L. Song, Phys. Plasmas 11, 4893 (2004).

(a)(i)

Open Access. This article is licensed under a Creative Commons Attribution 4.0 International License, which permits use, sharing, adaptation, distribution and reproduction in any medium or format, as long as you give appropriate credit to the original author(s) and the source, provide a link to the Creative Commons license, and indicate if changes were made. The images or other third party material in this article are included in the article $s$ Creative Commons license, unless indicated otherwise in a credit line to the material. If material is not included in the article's Creative Commons license and your intended use is not permitted by statutory regulation or exceeds the permitted use, you will need to obtain permission directly from the copyright holder. To view a copy of this license, visit: http://creativecommons.org/licenses/by/4.0/. 